\title{
ALTERAÇÕES NOS ATRIBUTOS DE FERTILIDADE EM SOLO ADUBADO COM COMPOSTO DE LIXO URBANO ${ }^{(1)}$
}

\author{
José Ricardo Mantovani ${ }^{(2)}$, Manoel Evaristo Ferreira ${ }^{(3)}$, \\ Mara Cristina Pessôa da Cruz $^{(4)}$ \& José Carlos Barbosa ${ }^{(5)}$
}

\begin{abstract}
RESUMO
O composto de lixo urbano é um adubo orgânico que vem sendo, com bastante freqüência, utilizado em áreas de produção de hortaliças. Assim, o objetivo deste trabalho foi avaliar o efeito da aplicação do composto de lixo urbano na fertilidade do solo, na produção de alface e no acúmulo de nutrientes nas plantas. O experimento foi realizado em casa de vegetação, em colunas de PVC, em delineamento em blocos ao acaso, com cinco tratamentos, doses de $0 ; 30 ; 60 ; 90$ e $120 \mathrm{t} \mathrm{ha}^{-1}$ de composto de lixo urbano e oito repetições. As colunas receberam solo das profundidades de 0-20 (tratado com composto de lixo), $20-40$ e 40-60 cm de um Argissolo, textura média, e uma muda de alface. Ao final do cultivo, colunas de quatro repetições de cada tratamento foram desmontadas e, nas demais colunas, fez-se um segundo cultivo de alface. A incorporação de composto de lixo urbano na profundidade de $0-20 \mathrm{~cm}$ melhorou a fertilidade do solo da própria camada em que foi aplicado e da camada de $20-40 \mathrm{~cm}$, mas não alterou as características da camada de 40-60 $\mathrm{cm}$. A adubação com composto de lixo urbano propiciou aumento do $\mathrm{pH}$ e dos teores de MO, P, K, Ca e Mg do solo, na camada de 0-20 cm, e de pH e Ca, na profundidade de $20-40 \mathrm{~cm}$. A melhora da fertilidade do solo com a aplicação de composto de lixo urbano acarretou aumento de produção de alface e provocou maior acúmulo de $\mathrm{P}, \mathrm{K}$ e Ca nas plantas.
\end{abstract}

Termos de indexação: adubação orgânica, pH, macronutrientes, alface.

(1) Projeto financiado pela FAPESP. Recebido para publicação em agosto de 2004 e aprovado em junho de 2005.

(2) Doutorando do Curso de Pós-Graduação em Agronomia (Produção Vegetal), Faculdade de Ciências Agrárias e Veterinárias, Universidade Estadual Paulista - FCAV/UNESP. Via de Acesso Prof. Paulo Donato Castellane s/n, CEP 14884-900 Jaboticabal (SP). Bolsista da FAPESP. E-mail: mantovanijr@yahoo.com

(3) Professor Titular do Departamento de Solos e Adubos, FCAV/UNESP. E-mail: evaristo@fcav.unesp.br

(4) Professora Assistente do Departamento de Solos e Adubos, FCAV/UNESP. E-mail: mcpcruz@fcav.unesp.br

(5) Professor Titular do Departamento de Ciências Exatas, FCAV/UNESP. E-mail: jcbarbosa@fcav.unesp.br 


\title{
SUMMARY: CHANGES IN FERTILITY ATTRIBUTES OF SOIL FERTILIZED WITH URBAN WASTE COMPOST
}

\begin{abstract}
Urban waste compost is an organic manure frequently used in horticultural areas. Thus, the objectives of this study were to evaluate the effects of urban waste compost on soil fertility, on lettuce production and on nutrient accumulation in plants. The experiment was carried out in a greenhouse using PVC columns. It was used a randomized block design with five treatments and eight replications. The treatments consisted of five urban waste compost rates $\left(0 ; 30 ; 60 ; 90\right.$; and $\left.120 \mathrm{th} \mathrm{h}^{-1}\right)$. The columns were filled up with soil from the 0-20 layer (treated with waste compost), 20-40 and 40-60 cm layer of a sandy-loam Alfisol, and one lettuce seedling was planted. At the end of the first cycle, the rings of the columns of four replicates of each treatment were taken apart and another lettuce cultivation was carried out in the remaining four replicate columns. The urban waste compost incorporated at a depth of 0-20 cm improved the soil fertility in this layer and of the 20-40 cm layer, but caused no alteration in the characteristics at the 40-60 cm layer. Fertilization with urban waste compost increased $\mathrm{pH}, \mathrm{OM}, \mathrm{P}, \mathrm{K}, \mathrm{Ca}$, and $\mathrm{Mg}$ in the $0-20 \mathrm{~cm}$ layer and $\mathrm{pH}$ and $\mathrm{Ca}$ at a depth of 20-40 cm. The improvement of soil fertility with the urban waste compost application increased the lettuce yield and $P, K$ and $C a$ accumulation by plants.
\end{abstract}

Index terms: organic fertilization, $p H$, macronutrients, lettuce.

\section{INTRODUÇÃO}

A utilização do composto de lixo urbano como adubo orgânico propicia reciclagem de nutrientes e melhoria das características físicas, químicas e biológicas do solo (Berton \& Valadares, 1991; He et al., 1992; Giusquiani et al., 1995). Em relação à fertilidade do solo, há relatos de aumentos do valor de $\mathrm{pH}$ e dos teores de MO, P, K, Ca e Mg (Mazur et al., 1983a; Alves et al., 1999; Abreu Jr. et al., 2000; Abreu Jr. et al., 2002; Oliveira et al., 2002a). Os aumentos obtidos variam de acordo com o solo, tipo de experimento (campo ou casa de vegetação), composição química do composto, grau de maturação e quantidades aplicadas.

Mazur et al. (1983a) constataram, em um Latossolo Amarelo, aumento de $57 \%$ no teor de P disponível com a aplicação do equivalente a $30 \mathrm{t} \mathrm{ha}^{-1}$ de composto de lixo. Abreu Jr. et al. (2002) verificaram, em amostras de 21 solos ácidos, incrementos no teor de $\mathrm{P}$ disponível que variaram de 29 a $417 \%$ com a adição de $60 \mathrm{t} \mathrm{ha}^{-1}$ do adubo orgânico. Quanto ao $\mathrm{pH}$, os aumentos relatados para a camada arável (0-20 $\mathrm{cm}$ de profundidade) estão entre 0,7 e 1,8 unidade a cada $60 \mathrm{t} \mathrm{ha}^{-1}$ de composto de lixo aplicadas (Alves et al., 1999; Abreu Jr. et al., 2000; Oliveira et al., 2002a). Wong et al. (1998) verificaram que, entre vários adubos orgânicos, o composto de lixo foi o que apresentou o maior efeito corretivo. Segundo Abreu Jr. et al. (2000), uma aplicação de $60 \mathrm{t} \mathrm{ha}^{-1}$ de composto de lixo, em condições de campo, tem efeito semelhante ao da adição de $2 \mathrm{t}^{\text {ha-1 }}{ }^{-1}$ de calcário.

A composição química do composto de lixo é bastante variável e, dentre os nutrientes presentes no adubo orgânico, o Ca é o que está em maiores concentrações (Berton \& Valadares, 1991). Cravo et al. (1998) obtiveram, para compostos de diferentes locais do Brasil, as seguintes concentrações, em $\mathrm{g} \mathrm{kg}^{-1}$ : 160 a 317 de MO; 93 a 275 de C; 8 a 15 de N; 2 a 4 de $\mathrm{P} ; 3$ a 11 de $\mathrm{K} ; 18$ a 36 de $\mathrm{Ca}$; e 2 a $5 \mathrm{de} \mathrm{Mg}$.

Além dos efeitos no solo, o uso de composto de lixo urbano pode propiciar aumento de produção e maior acúmulo de nutrientes nas plantas (Alves et al., 1999; Costa et al., 2001).

O objetivo deste trabalho foi avaliar o efeito do composto de lixo urbano na fertilidade do solo, na produção de alface e no acúmulo de nutrientes nas plantas.

\section{MATERIAL E MÉTODOS}

$\mathrm{O}$ experimento foi realizado em casa de vegetação, em colunas de PVC de $20 \mathrm{~cm}$ de diâmetro, no período de setembro de 2001 a janeiro de 2002 . Foram utilizadas amostras de um Argissolo Vermelho-Amarelo distrófico, coletadas em uma área de pastagem, nas profundidades de $0-20,20-40$ e 40-60 cm. Os resultados das análises de rotina e $\mathrm{Al}$ trocável (Raij et al., 1987), de $\mathrm{S}_{-} \mathrm{SO}_{4}{ }^{2-}$ (Cantarella \& Prochnow, 2001), de B (Abreu et al., 1994) e de Zn (Abreu et al., 2001) das amostras encontram-se no quadro 1. Em amostra da camada de $0-20 \mathrm{~cm}$, também foi feita análise granulométrica (Camargo et al., 1986), tendo-se obtido os seguintes teores de argila, areia e silte: $160 ; 770$ e $70 \mathrm{~g} \mathrm{~kg}^{-1}$, respectivamente.

Empregou-se o delineamento em blocos ao acaso, com cinco tratamentos e oito repetições. Os 
Quadro 1. Caracterização química do solo utilizado no experimento

\begin{tabular}{|c|c|c|c|c|c|c|c|c|c|c|c|c|c|}
\hline Profundidade & $P$ resina & MO & $\mathbf{p H ~} \mathrm{CaCl}_{2}$ & $\mathbf{K}$ & $\mathbf{C a}$ & $\mathbf{M g}$ & $\mathbf{H}+\mathbf{A l}$ & CTC & Al & $\mathbf{V}$ & $\mathrm{S}-S O_{4}{ }^{2-}$ & $\mathbf{B}$ & $\mathbf{Z n}$ \\
\hline $\mathrm{cm}$ & $\mathrm{mg} \mathrm{dm} \mathrm{m}^{-3}$ & $\mathrm{~g} \mathrm{dm}^{-3}$ & & $\ldots$ & 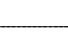 & $-\mathrm{mm}$ & $\mathrm{dm} \mathrm{m}^{-3}$ & 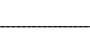 & - & $\%$ & $-\mathrm{m}$ & $\mathrm{g} \mathrm{dm} \mathrm{dm}^{-3}$ & - \\
\hline $00-20$ & 5 & 26 & 4,5 & 1,2 & 12 & 7 & 40 & 60 & 4 & 33 & 3 & 0,18 & 0,8 \\
\hline $20-40$ & 3 & 14 & 4,3 & 0,7 & 9 & 3 & 36 & 49 & 7 & 27 & 2 & 0,16 & 0,2 \\
\hline $40-60$ & 2 & 12 & 4,4 & 0,7 & 10 & 4 & 32 & 47 & 5 & 32 & 2 & 0,15 & 0,1 \\
\hline
\end{tabular}

tratamentos foram de doses equivalentes a $0 ; 30$; 60; 90 e $120 \mathrm{t} \mathrm{ha}^{-1}$ de composto de lixo urbano proveniente da Usina de Reciclagem de Lixo da cidade de Assis (SP). O adubo orgânico, analisado conforme descrito em Kiehl (1985), apresentou $60 \mathrm{~g} \mathrm{~kg}^{-1}$ de umidade, $\mathrm{pH}$ em $\mathrm{CaCl}_{2}=7,4$ e os seguintes resultados, na base seca: $\mathrm{C}=157 \mathrm{~g} \mathrm{~kg}^{-1}$; $\mathrm{N}=14 \mathrm{~g} \mathrm{~kg}^{-1} ; \mathrm{C} / \mathrm{N}=11 / 1 ; \mathrm{P}, \mathrm{K}, \mathrm{Ca} \mathrm{e} \mathrm{Mg}$,

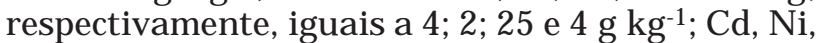
$\mathrm{Pb}, \mathrm{Cu}, \mathrm{Fe}, \mathrm{Mn}$ e $\mathrm{Zn}$, respectivamente, iguais a $<1$; $21 ; 80 ; 181 ; 26.852 ; 412$ e $544 \mathrm{mg} \mathrm{kg}^{-1}$.

Porções de $6,78 \mathrm{dm}^{3}$ de solo da camada superficial $(0-20 \mathrm{~cm})$, correspondentes a $8,345 \mathrm{~kg}$, receberam $\mathrm{CaCO}_{3}$ p.a. e $4 \mathrm{MgCO}_{3} \cdot \mathrm{Mg}(\mathrm{OH})_{2} \cdot 5 \mathrm{H}_{2} \mathrm{O}$ p.a., na proporção de Ca:Mg de 3:1, para elevar o V a $70 \%$, e o composto de lixo, de acordo com cada tratamento. As porções de solo foram transferidas para vasos de plástico com capacidade para $8 \mathrm{~L}$, umedecidas com água deionizada a $60 \%$ da capacidade de retenção, e permaneceram incubadas por 33 dias. Aos 23 dias de incubação, foi feita adubação mineral em todos os vasos, tendo sido adicionados, em mg vaso ${ }^{-1}: 550$ de P; 962 de K; 110 de S, e 2,75 de B, por meio de solução preparada a partir dos reagentes p.a. $\mathrm{KH}_{2} \mathrm{PO}_{4}, \mathrm{~K}_{2} \mathrm{SO}_{4}$ e $\mathrm{H}_{3} \mathrm{BO}_{3}$. Ao final da incubação, o solo de cada vaso foi seco ao ar e foi coletada amostra de $0,5 \mathrm{dm}^{3}$ para análise química de rotina (Raij et al., 1987).

As colunas de PVC foram compostas por três anéis de $20 \mathrm{~cm}$ de diâmetro, unidos por fita adesiva, e um "cap" (tampão) acoplado ao anel inferior. Cada anel apresentava $20 \mathrm{~cm}$ de altura, exceto o superior, que tinha $2 \mathrm{~cm}$ a mais para facilitar as irrigações. As paredes internas dos anéis de PVC receberam uma camada de resina líquida, sobre a qual foi espalhada uma mistura de areia grossa (diâmetro acima de 1,5 mm) e areia fina (diâmetro de 1,5 a $0,5 \mathrm{~mm}$ ), previamente lavadas, na proporção de $2: 1$ (v:v), para criar rugosidade e evitar o escoamento preferencial de água pelas paredes da coluna. Para a coleta de lixiviado, foi feito, próximo à base de cada tampão, um orifício lateral de $1 \mathrm{~cm}$ de diâmetro, e nele foi introduzida uma mangueira de plástico de $14 \mathrm{~cm}$ de comprimento, de tal maneira que cerca de $4 \mathrm{~cm}$ da mangueira ficavam do lado de fora do tampão. Para facilitar a drenagem, o fundo de cada tampão de PVC foi preenchido com esferas de isopor de $15 \mathrm{~mm}$ de diâmetro, e sobre elas foram adicionados cerca de $500 \mathrm{~mL}$ de areia fina lavada.

As colunas de PVC foram colocadas em suportes de ferro para facilitar as pesagens feitas para controle de umidade e foram preenchidas com auxílio de funil com haste longa, com $6,28 \mathrm{dm}^{3}$ de solo de cada profundidade, de modo que o solo da camada de 40-60 cm ocupou o anel inferior; o da camada de $20-40 \mathrm{~cm}$, o intermediário, e o da camada de $0-20 \mathrm{~cm}$ (tratada com carbonatos, composto de lixo e adubos) preencheu o anel superior. Em seguida, o solo de cada coluna foi umedecido a $60 \%$ da capacidade de retenção de água e recebeu, em 23-10-2001, uma muda de alface do grupo crespa, cultivar Verônica. A adubação nitrogenada foi feita por meio de solução de uréia p.a., de modo a aplicar $480 \mathrm{mg}$ de $\mathrm{N}$ por coluna, parcelados em quatro vezes: $20 \%$ no transplantio e 30,20 e $30 \%$ aos $10 ; 20$ e 30 dias do transplantio, respectivamente. Aos 32 dias após o transplantio, foi feita aplicação de $375 \mathrm{mg}$ de K por coluna, dos quais $183 \mathrm{mg}$ como $\mathrm{K}_{2} \mathrm{SO}_{4}$ p.a. e $192 \mathrm{mg}$ como $\mathrm{KCl}$ p.a.

Depois de 24 e 34 dias do transplantio, com o solo das colunas na umidade desejada $(60 \%$ da capacidade de retenção), adicionaram-se em cada coluna $500 \mathrm{~mL}$ de água deionizada a cada $30 \mathrm{~min}$ até completar $3 \mathrm{~L}$, o que equivale a uma precipitação de $32 \mathrm{~mm} \mathrm{~h}^{-1}$ durante $3 \mathrm{~h}$. Os lixiviados obtidos (cerca de $500 \mathrm{~mL} /$ coluna) foram coletados em vasilhas de plástico, tiveram seu volume medido e foi feita quantificação de K, por fotometria de chama, e de $\mathrm{Ca}$ e $\mathrm{Mg}$, por espectrofotometria de absorção atômica.

Aos 38 dias do transplantio, foi realizada a colheita das plantas, cortando-as rente à superfície do solo de cada coluna. A parte aérea das plantas foi lavada, seca em estufa a cerca de $65^{\circ} \mathrm{C}$ até peso constante, moída e submetida à digestão nítricoperclórica (Bataglia et al., 1983). Nos extratos, determinaram-se os teores de $\mathrm{P}$, por colorimetria; de $\mathrm{K}$, por fotometria de chama, e de $\mathrm{Ca}$ e $\mathrm{Mg}$, por espectrofotometria de absorção atômica.

Após a colheita das plantas, colunas de quatro repetições de cada tratamento foram desmontadas e, de cada anel, foi coletada amostra de solo para análise química de rotina (Raij et al., 1987). 
Nas colunas das outras quatro repetições, foi feito um segundo cultivo de alface. Para tanto, o solo foi reumedecido a $60 \%$ da capacidade de retenção de água e, em 10-12-2001, foi transplantada uma muda de alface do cultivar Verônica. A adubação nitrogenada foi idêntica à do primeiro cultivo, enquanto a adubação potássica de cobertura foi parcelada aos 10 e aos 30 dias do transplantio, tendo sido, na primeira adubação, aplicados, por meio de solução, $252 \mathrm{mg}$ de $\mathrm{K}$ por coluna, como $\mathrm{KH}_{2} \mathrm{PO}_{4}$ p.a. e, na segunda adubação, $498 \mathrm{mg}$ de $\mathrm{K}$ por coluna, sendo $60 \%$ como $\mathrm{K}_{2} \mathrm{SO}_{4}$ p.a. e $40 \%$ como $\mathrm{KCl}$ p.a. As demais etapas: lixiviações; colheita das plantas; coleta de amostra de solo por anel; análises de solo, planta e lixiviado foram feitas de maneira idêntica à citada no primeiro cultivo.

Os resultados obtidos foram submetidos à análise de variância, teste de comparação de médias (Tukey, a $5 \%$ ) e regressão polinomial. Na análise estatística dos resultados das análises de solo, ao final de cada cultivo, foi empregada análise de variância em parcelas subdivididas, tendo como parcelas as doses de composto de lixo e, nas subparcelas, as profundidades. Nas demais análises estatísticas, foram utilizadas análises de variância em delineamento em blocos ao acaso.

\section{RESULTADOS E DISCUSSÃO}

\section{Fertilidade do solo}

Nas amostras de solo coletadas após o período de incubação nos vasos, o composto de lixo urbano propiciou aumentos lineares no $\mathrm{pH} \mathrm{CaCl}_{2}$ e nos teores de $\mathrm{MO}, \mathrm{P}, \mathrm{K}^{+}, \mathrm{Ca}^{2+}$ e $\mathrm{Mg}^{2+}$, tendo sido constatados para esses atributos de fertilidade do solo acréscimos de 19, 28, 81, 27, 178 e $100 \%$, quando se comparou o tratamento-testemunha ao que recebeu a maior dose do adubo orgânico (Figura 1). Aumentos nos teores de macronutrientes e de MO do solo com a adição de composto de lixo também foram constatados por outros autores (Mazur et al., 1983a; Hernandes et al., 1992; Alves et al., 1999; Abreu Jr. et al., 2002).

Mesmo após os dois cultivos sucessivos de alface, pôde-se verificar aumento do $\mathrm{pH} \mathrm{CaCl} \mathrm{C}_{2}$ dos teores de $\mathrm{MO}, \mathrm{P}, \mathrm{Ca}^{2+} \mathrm{e} \mathrm{Mg}^{2+}$ na camada de 0-20 cm. Em relação ao $\mathrm{K}^{+}$, o efeito residual do adubo orgânico foi verificado apenas após o primeiro cultivo (Figura 1).

A adubação com composto de lixo na camada superficial $(0-20 \mathrm{~cm})$ proporcionou alterações na camada de $20-40 \mathrm{~cm}$, mas não na de $40-60 \mathrm{~cm}$. Na camada de $20-40 \mathrm{~cm}$, houve aumento do $\mathrm{pH}$ e dos teores de $\mathrm{Ca}^{2+}$ após cada cultivo de alface, e, ainda, dos de $\mathrm{K}^{+}$após o primeiro cultivo (Quadro 2). No caso do $\mathrm{pH}$ em $\mathrm{CaCl}_{2}$, o aumento foi de 0,1 unidade para cada $30 \mathrm{t} \mathrm{ha}^{-1}$ de adubo orgânico aplicado. Aumentos no pH do solo com a aplicação de composto de lixo urbano também foram observados por Mazur et al. (1983b), Wong et al. (1998), Abreu Jr. et al. (2000) e Oliveira et al. (2002a). Esse efeito no pH do solo, inclusive em profundidade, pode ser atribuído à presença de ânions orgânicos solúveis ( $\mathrm{R}-\mathrm{COO}^{-}$e $\mathrm{R}^{-} \mathrm{O}^{-}$) em resíduos orgânicos, que, ao serem liberados, podem adsorver $\mathrm{H}^{+}$da solução do solo por meio de reação de troca, envolvendo, principalmente, íons $\mathrm{Ca}^{2+}$. Quando oxidados, os compostos originados liberam $\mathrm{CO}_{2}$ e $\mathrm{H}_{2} \mathrm{O}$, diminuindo, assim, a acidez do solo (Pavan et al., 1997). Abreu Jr. et al. (2000) e Oliveira et al. (2002a) citam as seguintes teorias para explicar o efeito corretivo do composto de lixo: presença de humatos alcalinos no composto; produção de $\mathrm{OH}^{-}$, quando o oxigênio da solução do solo atua como receptor de elétrons provenientes da oxidação microbiana do carbono orgânico do resíduo; consumo de $\mathrm{H}^{+}$e complexação de $\mathrm{H}^{+}$e $\mathrm{Al}^{3+}$ pelo composto. Wong et al. (1998) verificaram que os aumentos no $\mathrm{pH}$ de solos tropicais devidos à aplicação de adubos orgânicos foram diretamente proporcionais à capacidade desses materiais em consumir $\mathrm{H}^{+}$, e que essa característica está relacionada com o total de bases $\left(\mathrm{Ca}^{2+}, \mathrm{Mg}^{2+}, \mathrm{K}^{+} \mathrm{e} \mathrm{Na}^{+}\right)$presente nos resíduos.

$\mathrm{O}$ aumento na disponibilidade de $\mathrm{P}$ deve-se à presença do nutriente no adubo orgânico e aos aumentos do valor de $\mathrm{pH}$ e do teor de matéria orgânica (Mazur et al., 1983a; Abreu Jr. et al., 2002). $\mathrm{O}$ aumento do $\mathrm{pH}$ do solo até próximo a 7,0 propicia maior disponibilidade de $\mathrm{P}$, uma vez que, em condições ácidas, ocorre reação do $\mathrm{H}_{2} \mathrm{PO}_{4}{ }^{-}$com as formas iônicas de $\mathrm{Fe}$ e $\mathrm{Al}$, formando compostos de baixa solubilidade, além de maior adsorção do ânion por óxidos de $\mathrm{Fe}$ e $\mathrm{Al}$ presentes na fase sólida. A matéria orgânica, por sua vez, bloqueia os sítios de adsorção em óxidos de $\mathrm{Fe}$ e de $\mathrm{Al}$ do solo, diminuindo a capacidade de adsorção do $\mathrm{H}_{2} \mathrm{PO}_{4}^{-}$(Novais \& Smyth, 1999).

Os teores de $\mathrm{K}^{+}, \mathrm{Ca}^{2+}$ e $\mathrm{Mg}^{2+}$ no solo da camada de $20-40 \mathrm{~cm}$ de profundidade, na condição inicial, foram de 0,7; 9 e $3 \mathrm{mmol}_{\mathrm{c}} \mathrm{dm}^{-3}$, respectivamente. Após um cultivo de alface, eles passaram para 1,5; 10 e $5 \mathrm{mmol}_{\mathrm{c}} \mathrm{dm}^{-3}$, no tratamento-testemunha, e para 1,$8 ; 14$ e $6 \mathrm{mmol}_{\mathrm{c}} \mathrm{dm}^{-3}$, no tratamento que recebeu $120 \mathrm{t} \mathrm{ha}^{-1}$ de composto de lixo (Figura 2). Portanto, em relação à condição inicial, houve, nessa profundidade, aumento de 114, 11 e $66 \%$ nos teores de $\mathrm{K}^{+}, \mathrm{Ca}^{2+}$ e $\mathrm{Mg}^{2+}$ trocáveis no tratamentotestemunha, e de 157,55 e $100 \%$ no que recebeu a maior dose do adubo orgânico. Ao final do segundo cultivo, os aumentos obtidos para os mesmos tratamentos foram de 71,10 e $67 \%$, e de 71,44 e $100 \%$, respectivamente. Esses resultados evidenciam que houve lixiviação de $\mathrm{K}^{+}, \mathrm{Ca}^{2+} \mathrm{e} \mathrm{Mg}^{2+}$ da camada superficial $(0-20 \mathrm{~cm})$ para a de $20-40 \mathrm{~cm}$. Observou-se, ainda, que as perdas por lixiviação aumentaram com as doses de composto de lixo. 


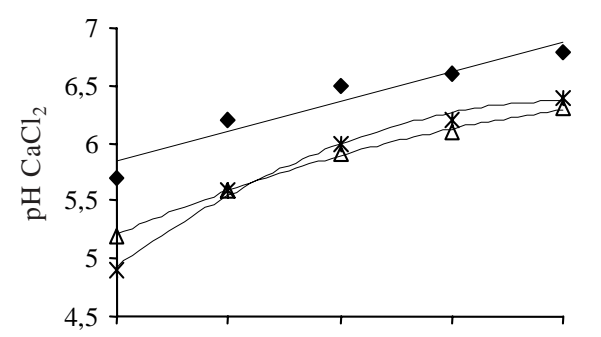

após incubação: $\hat{\mathrm{y}}=0,009 \mathrm{x}+5,840 ; \mathrm{R}^{2}=0,942 * *$

após $1^{\circ}$ cultivo: $\hat{y}=-0,00005 x^{2}+0,015 x+5,169 ; R^{2}=0,996^{* *}$

após $2^{\circ}$ cultivo: $\hat{y}=-0,0001 x^{2}+0,023 x+4,939 ; R^{2}=0,990 * *$

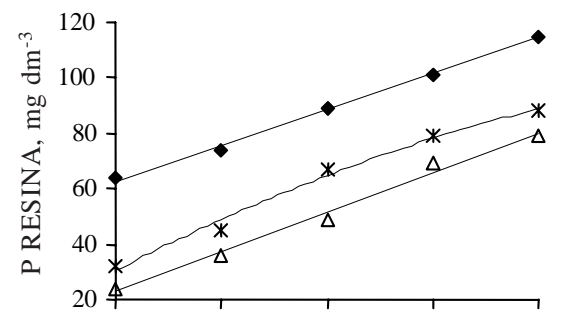

após incubação: $\hat{y}=0,430 x+62,800 ; R^{2}=0,997 * *$ após $1^{\circ}$ cultivo: $\hat{\mathrm{y}}=0,474 \mathrm{x}+22,900 ; \mathrm{R}^{2}=0,990^{* *}$ após $2^{\circ}$ cultivo: $\hat{y}=-0,0114 x^{2}+0,658 x+30,200 ; R^{2}=0,990 * *$

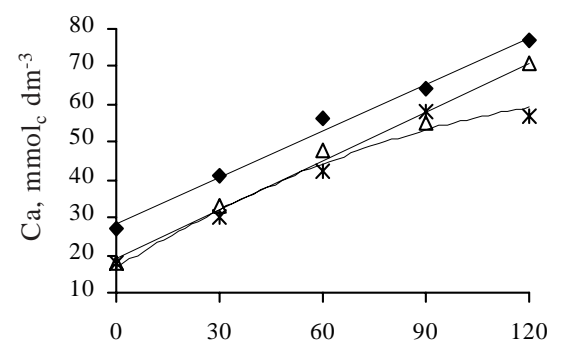

após incubação: $\hat{y}=0,410 x+28,400 ; R^{2}=0,991^{* *}$ após $1^{\circ}$ cultivo: $\hat{\mathrm{y}}=0,423 \mathrm{x}+19,500 ; \mathrm{R}^{2}=0,989 * *$ após $2^{\circ}$ cultivo: $\hat{y}=-0,0017 x^{2}+0,569 x+16,220 ; R^{2}=0,966 * *$
- após incubação $\triangle$ após $1^{\circ}$ cultivo * após $2^{\circ}$ cultivo

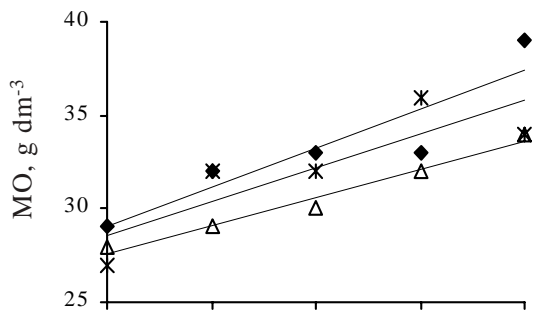

após incubação: $\hat{y}=0,070 x+29,000 ; R^{2}=0,835^{* *}$ após $1^{\circ}$ cultivo: $\hat{\mathrm{y}}=0,054 \mathrm{x}+27,600 ; \mathrm{R}^{2}=0,970^{* *}$ após $2^{\circ}$ cultivo: $\hat{y}=0,059 x+28,750 ; R^{2}=0,728^{* *}$

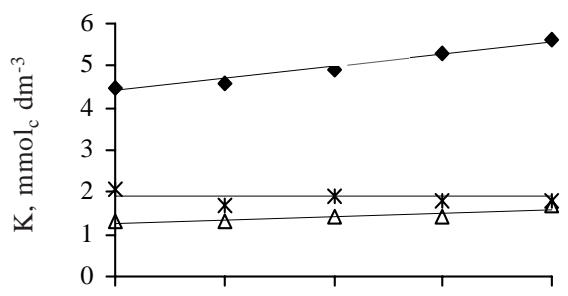

após incubação: $\hat{y}=0,010 x+4,400 ; R^{2}=0,969 * *$ após $1^{\circ}$ cultivo: $\hat{\mathrm{y}}=0,003 \mathrm{x}+1,205 ; \mathrm{R}^{2}=0,849^{* *}$ após $2^{\circ}$ cultivo: $\hat{y}=1,9$

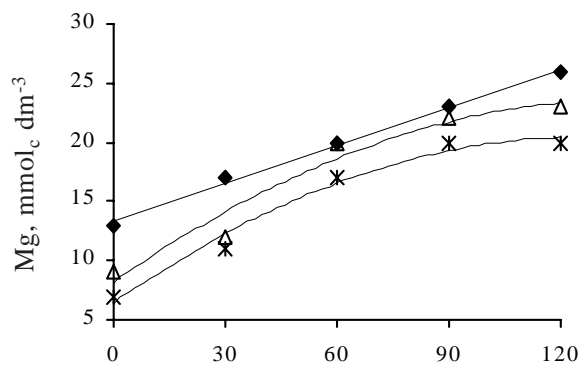

COMPOSTO DE LIXO, $\mathrm{t} \mathrm{ha}^{-1}$

após incubação: $\hat{y}=0,107 x+13,400 ; R^{2}=0,996^{* *}$

após $1^{\circ}$ cultivo: $\hat{y}=-0,0008 x^{2}+0,232 x+7,814 ; R^{2}=0,970 * *$ após $2^{\circ}$ cultivo: $\hat{y}=-0,0008 x^{2}+0,214 x+6,207 ; R^{2}=0,981 * *$

Figura 1. Efeito do composto de lixo urbano nas características químicas do solo.

Quadro 2. Estimativa dos valores de pH, teores de $\mathrm{K}$ e Ca no solo, da camada de $20-40 \mathrm{~cm}$ ( $\hat{\mathrm{y}}$ ), após cada cultivo de alface, considerando as doses de composto de lixo urbano (x)

\begin{tabular}{cccc}
\hline Variável dependente $(\mathbf{y})$ & Cultivo & Equação & $\mathbf{R}^{\mathbf{2}}$ \\
\hline $\mathrm{pH} \mathrm{CaCl}{ }_{2}$ & $1^{\circ}$ & $\hat{\mathrm{y}}=0,003 \mathrm{x}+4,410$ & $0,920^{* *}$ \\
& $2^{\circ}$ & $\hat{\mathrm{y}}=0,003 \mathrm{x}+4,320$ & $0,990^{* *}$ \\
$\mathrm{~K}\left(\mathrm{mmol}_{\mathrm{c}} \mathrm{dm}^{-3}\right)$ & $1^{\circ}$ & $\hat{\mathrm{y}}=0,003 \mathrm{x}+1,390$ & $0,887^{* *}$ \\
$\mathrm{Ca}\left(\mathrm{mmol}_{\mathrm{c}} \mathrm{dm}^{-3}\right)$ & $1^{\circ}$ & $\hat{\mathrm{y}}=0,045 \mathrm{x}+8,900$ & $0,975^{* *}$ \\
& $2^{\circ}$ & $\hat{\mathrm{y}}=0,038 \mathrm{x}+9,100$ & $0,948^{* *}$ \\
\hline
\end{tabular}

As perdas por lixiviação e a absorção dos nutrientes pelas plantas explicam a diminuição nos teores de $\mathrm{K}^{+}, \mathrm{Ca}^{2+}$ e $\mathrm{Mg}^{2+}$, no solo da camada superficial com os cultivos. Em relação à camada de 40-60 $\mathrm{cm}$ de profundidade, os teores iniciais de $\mathrm{K}^{+}, \mathrm{Ca}^{2+} \mathrm{e} \mathrm{Mg}^{2+}$ foram de 0,$7 ; 10$ e $4 \mathrm{mmol}_{\mathrm{c}} \mathrm{dm}^{-3}$, respectivamente, e praticamente não se alteraram ao final de cada cultivo de alface (Figura 2). Os nutrientes nas camadas de $20-40$ e de $40-60 \mathrm{~cm}$ dificilmente seriam absorvidos pelas plantas por estarem fora do alcance das raízes, pois verificouse, por ocasião da separação dos anéis das colunas, que o sistema radicular da alface restringiu-se aos $20 \mathrm{~cm}$ superficiais. 

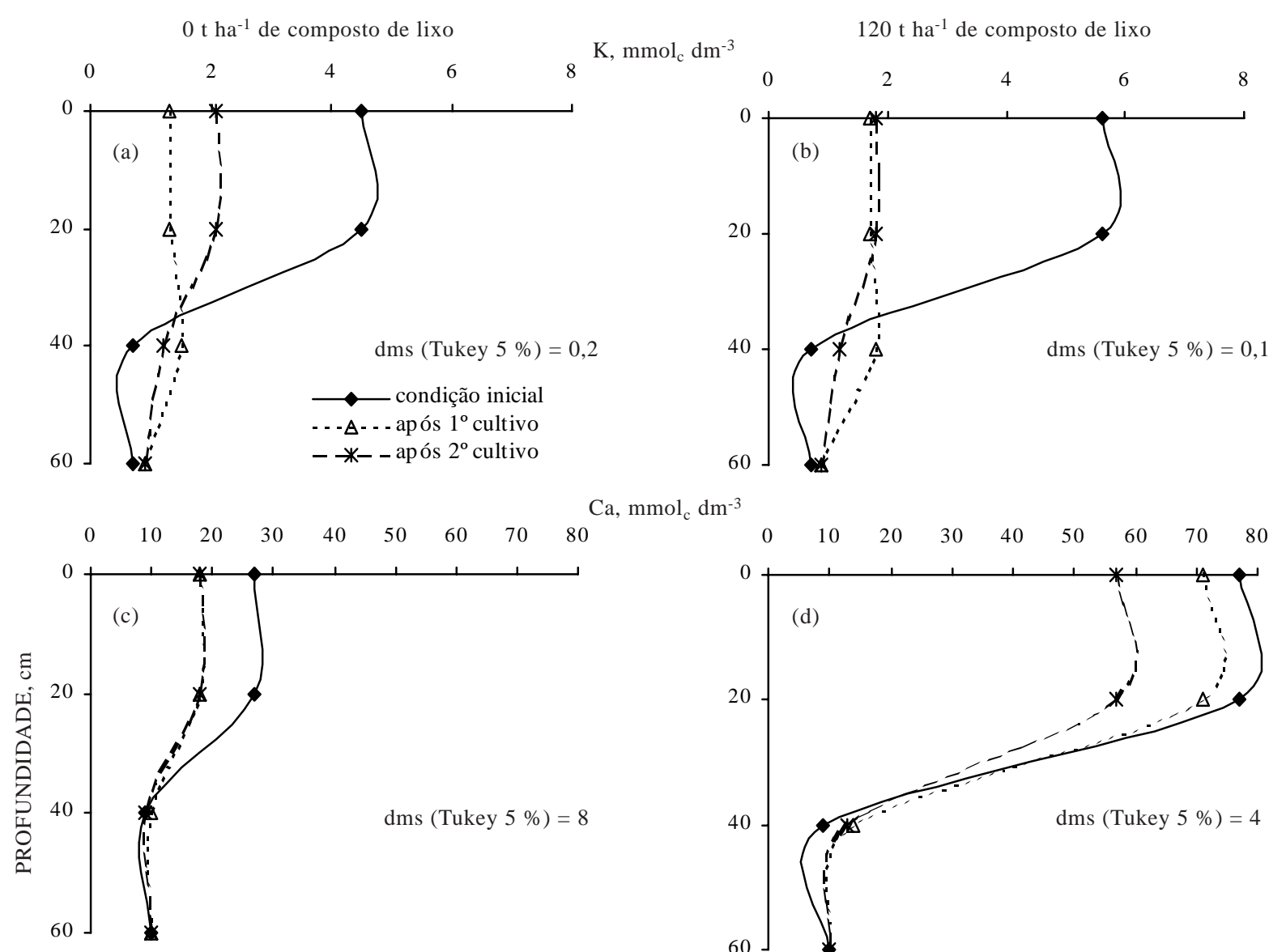

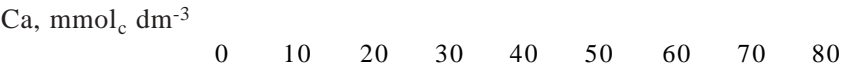
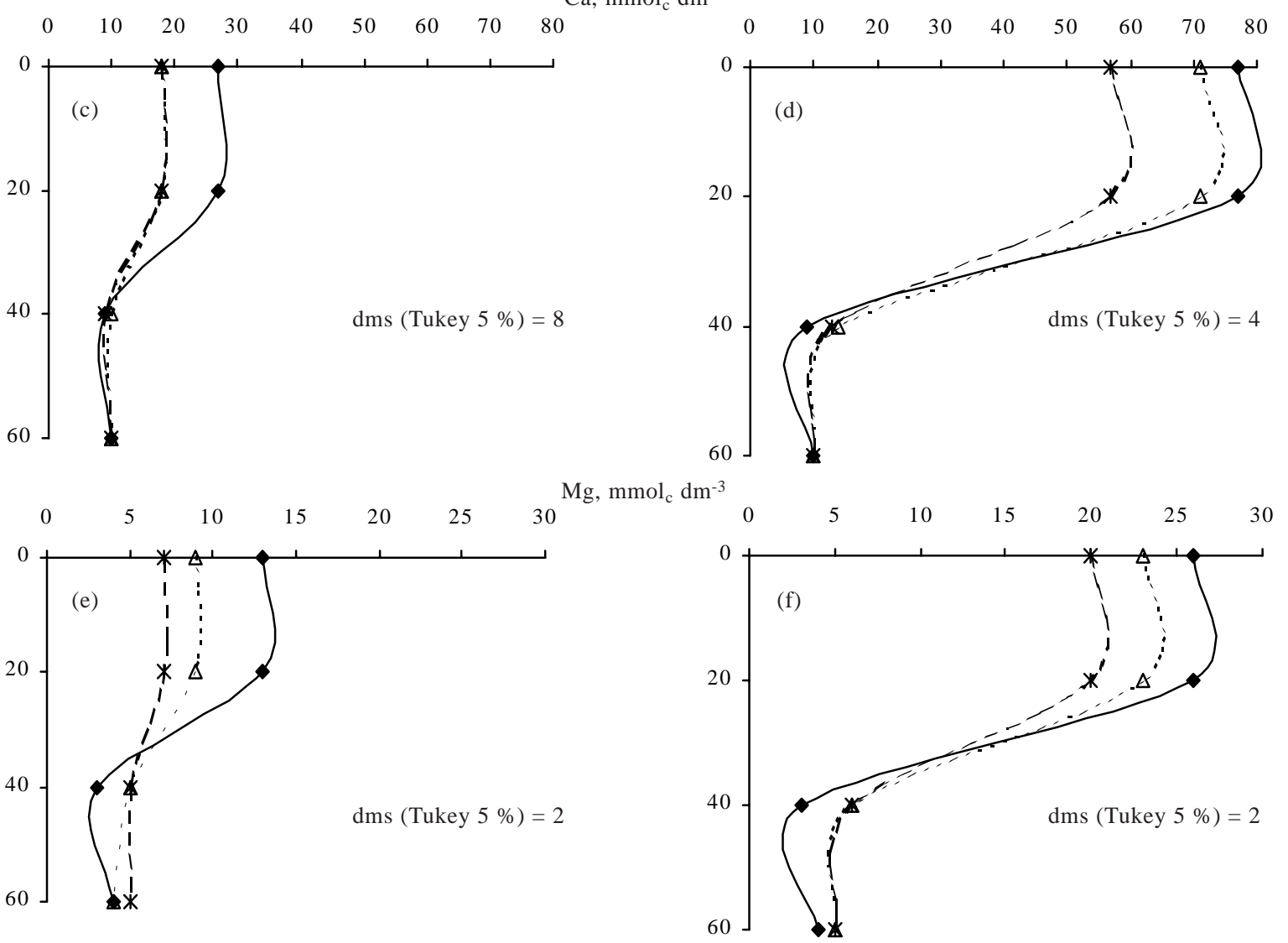

Figura 2. Teores de K, Ca e Mg no solo, de acordo com a profundidade e doses de composto de lixo urbano.

Moraes (1991) constatou perdas de $\mathrm{K}^{+}, \mathrm{Ca}^{2+} \mathrm{e}$ $\mathrm{Mg}^{2+}$ por lixiviação em Latossolo Vermelho, com o $\mathrm{NO}_{3}{ }^{-}$tendo participação importante no processo.

As quantidades de $\mathrm{K}^{+}$no lixiviado das colunas em que foram realizados um e dois cultivos de alface não foram afetadas significativamente nem apresentaram tendência definida com a adubação com composto de lixo, tendo as quantidades médias atingido de 9 a $24 \mathrm{mg}$ nas colunas com um e dois cultivos, respectivamente. Em contrapartida, as quantidades de $\mathrm{Ca}^{2+}$ e de $\mathrm{Mg}^{2+}$ no lixiviado aumentaram linearmente com as doses de composto de lixo e variaram de 53 a $104 \mathrm{mg}$ e de 11 a $30 \mathrm{mg}$ nas colunas com um cultivo de alface e de 175 a $283 \mathrm{mg}$ e de 43 a $77 \mathrm{mg}$ nas colunas com dois cultivos, para $\mathrm{Ca}^{2+} \mathrm{e} \mathrm{Mg}^{2+}$, respectivamente. As equações de regressão obtidas para $\mathrm{Ca}^{2+} \mathrm{e} \mathrm{Mg}^{2+}$ no lixiviado das colunas que receberam um cultivo foram: $\hat{y}_{\mathrm{Ca}}=$ $0,424 \mathrm{x}+53,050, \mathrm{R}^{2}=0,890 * * ; \hat{y}_{\mathrm{Mg}}=0,163 \mathrm{x}+10,600$, $\mathrm{R}^{2}=0,899^{* *} \mathrm{e}$, nas que receberam dois cultivos, foram: $\hat{\mathrm{y}}_{\mathrm{Ca}}=0,899 \mathrm{x}+175,200, \mathrm{R}^{2}=0,973^{* *} ; \hat{\mathrm{y}}_{\mathrm{Mg}}=$ $0,286 \mathrm{x}+43,000, \mathrm{R}^{2}=0,979^{* *}$, em que ye e a quantidade do nutriente lixiviada $\left(\mathrm{Ca}^{2+}\right.$ ou $\left.\mathrm{Mg}^{2+}\right) \mathrm{em}$ 
mg coluna ${ }^{-1}$, e x é a quantidade de composto de lixo aplicada em t ha ${ }^{-1}$. Anjos \& Mattiazzo (2000) verificaram, em dois Latossolos, aumento nas quantidades de $\mathrm{K}^{+}, \mathrm{Ca}^{2+}$ e $\mathrm{Mg}^{2+}$ no lixiviado com a aplicação de $78 \mathrm{t} \mathrm{ha}^{-1}$ de biossólido, sendo as de $\mathrm{Ca}^{2+}$ maiores do que as de $\mathrm{Mg}^{2+}$ e $\mathrm{K}^{+}$. Oliveira et al. (2002b), em ensaio utilizando lisímetros, também constataram que as quantidades de $\mathrm{Ca}^{2+}$ presentes no lixiviado excederam as de $\mathrm{Mg}^{2+}$ e de $\mathrm{K}^{+}$.

\section{Produção de alface e acúmulo de nutrientes}

O composto de lixo teve efeito quadrático na produção de matéria fresca de alface no primeiro e no segundo cultivos: no primeiro, houve aumento a partir da dose estimada de $38 \mathrm{t} \mathrm{ha}^{-1} \mathrm{e}$, no segundo, o aumento de matéria fresca ocorreu até $80 \mathrm{t} \mathrm{ha}^{-1}$ (Figura 3). No primeiro cultivo, o aumento de produção com a aplicação de $120 \mathrm{t}^{\mathrm{h}} \mathrm{a}^{-1}$ de composto de lixo foi de $8 \%$ em relação ao tratamentotestemunha. No segundo cultivo, esse aumento foi de $17 \%$ e chegou a $22 \%$, quando se comparou a produção máxima teórica com a obtida no tratamento que não recebeu adubação orgânica. Em relação à matéria seca, no primeiro cultivo, não foi observada variação significativa com as doses de composto de lixo, tendo sido a produção média de 22,06 g planta $^{-1}$, e, no segundo cultivo, verificou-se efeito quadrático, com aumento de produção de até $77 \mathrm{t} \mathrm{ha}^{-1}$ de composto de lixo. Costa et al. (1994) verificaram, em experimento em vasos empregando dois solos argilosos com acidez elevada e baixa fertilidade, aumentos na produção de matéria fresca e de matéria seca de alface até à dose estimada de $20 \mathrm{t} \mathrm{ha}^{-1}$ de composto de lixo urbano. Costa et al. (2001), em experimento de campo, observaram que a aplicação de composto de lixo urbano propiciou aumentos lineares na produção de matéria fresca e de matéria seca de alface em dois cultivos sucessivos. No terceiro cultivo, os autores não observaram efeito significativo na produção. Mantovani et al. (2003), em experimento em casa de vegetação, constataram que a aplicação de vermicomposto de lixo urbano, em doses acima de $50 \mathrm{t} \mathrm{ha}^{-1}$, limitou a produção de matéria seca de alface, tanto em solo arenoso quanto em solo argiloso.

No primeiro cultivo, as quantidades acumuladas de $\mathrm{P}, \mathrm{K}$ e Ca na parte aérea das plantas aumentaram linearmente com a aplicação de composto de lixo. No segundo cultivo, houve aumento linear para $\mathrm{P} \mathrm{e}$ efeito quadrático para $\mathrm{Ke} \mathrm{Ca}$, sendo constatado para esses nutrientes aumento até às doses estimadas de 90 e $100 \mathrm{t} \mathrm{ha}^{-1}$ de composto de lixo, respectivamente (Quadro 3). As quantidades acumuladas de $\mathrm{Mg}$ nas plantas não foram afetadas pela adubação orgânica, atingindo médias para o primeiro e segundo cultivo de 32 e $46 \mathrm{mg} \mathrm{planta}^{-1}$, respectivamente. Alves et al. (1999) também verificaram aumento nas quantidades acumuladas de $\mathrm{K}$, Ca e Mg na parte aérea de plantas de milho com a aplicação de composto de lixo urbano.
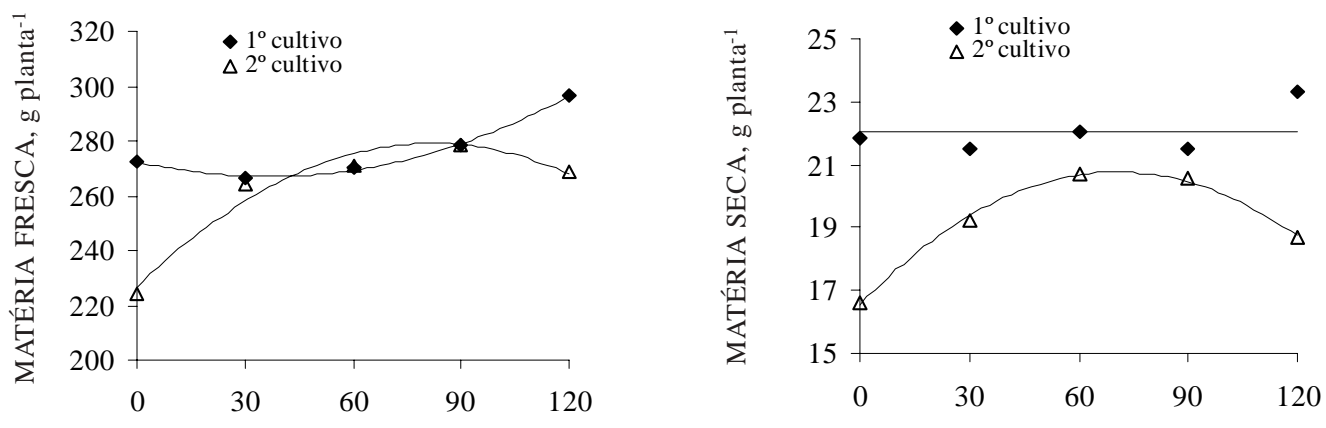

COMPOSTO DE LIXO, $\mathrm{t} \mathrm{ha}^{-1}$

$1^{\circ}$ cultivo: $\hat{y}=0,004 x^{2}-0,303 x+272,331 ; R^{2}=0,998 * *$

$1^{\circ}$ cultivo: $\hat{\mathrm{y}}=22,06$

$2^{\circ}$ cultivo: $\hat{y}=-0,008 x^{2}+1,284 x+226,525 ; R^{2}=0,926 * *$

$2^{\circ}$ cultivo: $\hat{y}=-0,0008 x^{2}+0,123 x+16,424 ; R^{2}=0,998^{* *}$

Figura 3. Produção de matéria fresca e de matéria seca de alface em cada cultivo, de acordo com a adubação com composto de lixo urbano.

Quadro 3. Valores estimados de nutrientes acumulados (y, $\left.\mathrm{mg}_{\text {planta }}{ }^{-1}\right)$ na parte aérea de alface, considerando as doses de composto de lixo urbano $\left(x, t\right.$ ha $\left.^{-1}\right)$, em cada cultivo

\begin{tabular}{cccc}
\hline Variável dependente $(\mathbf{y})$ & Cultivo & Equação & $\mathbf{R}^{2}$ \\
\hline P (mg/planta) & $1^{\circ}$ & $\hat{y}=0,035 \mathrm{x}+41,025$ & $0,813^{* *}$ \\
& $2^{\circ}$ & $\hat{y}=0,057 \mathrm{x}+49,800$ & $0,756^{*}$ \\
K (mg/planta) & $1^{\circ}$ & $\hat{y}=1,708 \mathrm{x}+709,000$ & $0,982^{* *}$ \\
Ca (mg/planta) & $2^{\circ}$ & $\hat{y}=-0,023 \mathrm{x}^{2}+4,087 \mathrm{x}+428,386$ & $0,972^{* *}$ \\
& $1^{\circ}$ & $\hat{y}=0,337 \mathrm{x}+132,975$ & $0,968^{* *}$ \\
& $2^{\circ}$ & $\hat{y}=-0,006 \mathrm{x}^{2}+1,196 \mathrm{x}+104,579$ & $0,973^{* *}$ \\
\hline
\end{tabular}




\section{CONCLUSÕES}

1. A incorporação de composto de lixo urbano na profundidade de $0-20 \mathrm{~cm}$ melhorou a fertilidade do solo da própria camada em que foi aplicado e da camada de $20-40 \mathrm{~cm}$, mas não alterou as características da camada de 40-60 cm.

2. A adubação com composto de lixo urbano propiciou aumento do $\mathrm{pH}$ e dos teores de $\mathrm{MO}, \mathrm{P}, \mathrm{K}$, Ca e Mg na camada de $0-20 \mathrm{~cm}$, bem como de $\mathrm{pH}$ e Ca na profundidade de $20-40 \mathrm{~cm}$.

3. A melhora da fertilidade do solo com a aplicação de composto de lixo urbano refletiu-se no aumento de produção de alface e provocou maior acúmulo de $\mathrm{P}, \mathrm{K}$ e Ca nas plantas.

\section{LITERATURA CITADA}

ABREU, C.A.; ABREU, M.F.; RAIJ, B. van; BATAGLIA, O.C. \& ANDRADE, J.C. Extraction of boron from soil by microwave heating for ICP-AES determination. Comm. Soil Sci. Plant Anal., 25:3321-3333, 1994

ABREU, C.A.; ABREU, M.F. \& ANDRADE, J.C. Determinação de cobre, ferro, manganês, zinco, cádmio, cromo, níquel e chumbo em solos usando a solução de DTPA em pH 7,3. In: RAIJ, B. van; ANDRADE, J.C.; CANTARELLA, H. \& QUAGGIO, J.A., eds. Análise química para avaliação da fertilidade de solos tropicais. Campinas, Instituto Agronômico de Campinas, 2001. p.240-250.

ABREU Jr., C.H.; MURAOKA, T.; LAVORANTE, A.F. \& ALVAREZ, V.F.C. Condutividade elétrica, reação do solo e acidez potencial em solos adubados com composto de lixo. R. Bras. Ci. Solo, 24:635-647, 2000

ABREU Jr., C.H.; MURAOKA, T. \& OLIVEIRA, F.C. Carbono, nitrogênio, fósforo e enxofre em solos tratados com composto de lixo urbano. R. Bras. Ci. Solo, 26:769-780, 2002.

ALVES, W.L.; MELO, W.J. \& FERREIRA, M.E. Efeito do composto de lixo urbano em um solo arenoso e em plantas de sorgo. R. Bras. Ci. Solo, 23:729-736, 1999.

ANJOS, A.R.M. \& MATTIAZZO, M.E. Lixiviação de íons inorgânicos em solos repetidamente tratados com biossólido. R. Bras. Ci. Solo, 24:927-938, 2000.

BATAGLIA, O.C.; FURLANI, A.M.C.; TEIXEIRA, J.P.F.; FURLANI, P.R. \& GALLO, J.R. Métodos de análise química de plantas. Campinas, Instituto Agronômicode Campinas, 1983. 48p. (Boletim Técnico, 78)

BERTON, R.S. \& VALADARES, J.M.A.S. Potencial agrícola do composto de lixo urbano no Estado de São Paulo. O Agronômico, 43:87-93, 1991.

CAMARGO, O.A.; MONIZ, A.C.; JORGE, J.A. \& VALADARES, J.M.A.S. Métodos de análise química, mineralógica e física de solos do Instituto Agronômico de Campinas. Campinas, Instituto Agronômico de Campinas, 1986. 94p. (Boletim Técnico, 106)

CANTARELLA, H. \& PROCHNOW, L.I. Determinação de sulfato em solos. In: RAIJ, B. van; ANDRADE, J.C.; CANTARELLA, H. \& QUAGGIO, J.A., eds. Análise química para avaliação da fertilidade de solos tropicais. Campinas, Instituto Agronômico de Campinas, 2001. p.225-230.
COSTA, C.A.; CASALI, V.W.D.; LOURES, E.G.; CECON, P.R. \& JORDÃO, C.P. Teor de metais pesados em alface (Lactuca sativa L.) adubada com composto orgânico de lixo urbano. R. Ceres, 41:629-640, 1994.

COSTA, C.A.; CASALI, V.W.D.; RUIZ, H.A.; JORDÃO, C.P. \& CECON, P.R. Teor de metais pesados e produção de alface adubada com composto de lixo urbano. Hortic. Bras., 19:10$16,2001$.

CRAVO, M.S.; MURAOKA, T. \& GINÉ, M.F. Caracterização química de compostos de lixo urbano de algumas usinas brasileiras. R. Bras. Ci. Solo, 22:547-553, 1998.

GIUSQUIANI, P.L.; PAGLIAI, M.; GIGLIOTTI, G.; BUSINELLI, D. \& BENETTI, A. Urban waste compost: effects on physical, chemical and biochemical soil properties. J. Environ. Qual., 24:175-182, 1995.

HE, X.T.; TRAINA, S.J. \& LOGAN, T.J. Chemical properties of municipal solid waste composts. J. Environ. Qual., 21:318329, 1992.

HERNANDEZ, T.; GARCIA, C.; COSTA, F.; VALERO, J.A. \& AYUSO, M. Utilizacion de residuos urbanos como fertilizantes organicos. Suelo Planta, 2:373-383, 1992.

KIEHL, E.J. Fertilizantes orgânicos. São Paulo, Agronômica Ceres, 1985. 492p.

MANTOVANI, J.R.; FERREIRA, M.E.; CRUZ, M.C.P.; CHIBA, M.K. \& BRAZ, L.T. Calagem e adubação com vermicomposto de lixo urbano na produção e nos teores de metais pesados em alface. Hortic. Bras., 21:494-500, 2003.

MAZUR, N.; SANTOS, G.A. \& VELLOSO, A.C.X. Efeito do composto de resíduo urbano na disponibilidade de fósforo em solo ácido. R. Bras. Ci. Solo, 7:153-156, 1983a.

MAZUR, N.; VELLOSO, A.C.X. \& SANTOS, G.A. Efeito do composto de resíduo urbano no $\mathrm{pH}$ e alumínio trocável em solo ácido. R. Bras. Ci. Solo, 7:157-159, 1983b.

MORAES, J.F.V. Movimento de nutrientes em Latossolo Vermelho-Escuro. Pesq. Agropec. Bras., 26:85-97, 1991

NOVAIS, R.F. \& SMYTH, T.J. Fósforo em solo e planta em condições tropicais. Viçosa, Universidade Federal de Viçosa, 1999. $399 \mathrm{p}$

OLIVEIRA, F.C.; MATTIAZZO, M.E.; MARCIANO, C.R. \& ABREU Jr., C.H. Alterações em atributos químicos de um Latossolo pela aplicação de composto de lixo urbano. Pesq. Agropec. Bras., 37:529-538, 2002a.

OLIVEIRA, M.W.; TRIVELIN, P.C.O.; BOARETTO, A.E.; MURAOKA, T. \& MORTATTI, J. Leaching of nitrogen, potassium, calcium and magnesium in sandy soil cultivated with sugarcane. Pesq. Agropec. Bras., 37:861-868, 2002b.

PAVAN, M.A.; CHAVES, J.C.D.; SIQUEIRA, R. \& ANDROCIOLI FILHO, A. Cultura do cafeeiro: O sistema de plantio adensado e a melhoria da fertilidade do solo. Inf. Agron., 80:1-7, 1997.

RAIJ, B. van; QUAGGIO, J.A.; CANTARELLA, H.; FERREIRA, M.E.; LOPES, A.S. \& BATAGLIA, O.C. Análise química do solo para fins de fertilidade. Campinas, Fundação Cargill, 1987. $170 \mathrm{p}$

WONG, M.T.F.; NORTCLIFF, S. \& SWIFT, R.S. Method for determining the acid ameliorating capacity of plant residue compost, urban waste compost, farmyard manure and peat applied to tropical soils. Comm. Soil Sci. Plant Anal., 29:2927-2937, 1998 